\title{
Molecular Cytogenetic Identification of a Wheat-Thinopyrum ponticum Substitution Line with Stripe Rust Resistance
}

\author{
Q. Mo**, C.Y. WAnG**, C.H. Chen, Y.J. Wang, H. Zhang, X.L. LiU and W.Q. JI* \\ State Key Laboratory of Crop Stress Biology for Arid Areas, College of Agronomy, \\ Northwest A\&F University, Yangling, Shaanxi 712100, China \\ (Received 13 October 2016; Accepted 3 March 2017; \\ Communicated by M. Molnár-Láng)
}

\begin{abstract}
Thinopyrum ponticum $(2 n=10 x=70)$ has donated rust resistance genes to protect wheat from this fungal disease. In the present study, the line ES-7, derived from the progeny of the crosses between common wheat cultivar Abbondanza and Triticum aestivum-Th. ponticum partial amphiploid line Xiaoyan784, was characterized by cytological, fluorescence in situ hybridization (FISH), genomic in situ hybridization (GISH) and EST-STS marker techniques. Cytological observations revealed that the configuration of ES-7 was $2 n=42=21$ II. GISH and FISH results showed that ES-7 had two St chromosomes and lacked 5A chromosomes compared to common wheat. The 4A chromosome of ES-7 had small alterations from common wheat. Two EST-SSR markers BE482522 and BG262826, specific to Th. ponticum and tetraploid Pseudoroegneria spicata $(2 n=4 x=28)$, locate on the homoeologous group 5 chromosomes of wheat, could amplify polymorphic bands in ES-7. It was suggested that the introduced St chromosomes belonged to homoeologous group 5, that is, ES-7 was a 5St (5A) disomic substitution line. Furthermore, ES-7 showed highly resistance to mixed stripe rust races of CYR32 and CYR33 in adult stages, which was possibly inherited from Th. ponticum. Thus, ES-7 can be used for wheat stripe rust resistance breeding program.
\end{abstract}

Keywords: wheat, Thinopyrum ponticum, disomic substitution line, stripe rust resistance, in situ hybridization, EST-STS markers

\section{Introduction}

The stripe rust, caused by Puccinia striiformis f. sp. tritici (Pst), generally impairs annual yield. Although stripe rusts generally produce fewer losses than stem rust and leaf rust (Huerta-Espino et al. 2011), it is widespread due to high variability of physiological race. Even though nearly 70 stripe rust resistance (Yr) genes have been cataloged and mapped to specific chromosomes, and plenty of addition lines, substitution lines, translocation lines and introgression lines have been developed between wheat and its relatives, such as Secale and Aegilops (Mcintosh 2003), many of the resistance genes may have been overwhelmed by newly emerged stripe rust races. Hence, the continuous production of resistance resources and characterization of novel resistance genes are essential to wheat breeding.

\footnotetext{
*Corresponding author; E-mail: jiwanquan2008@126.com; Phone: +86-29-87081319; Fax: +86-29-87081319 **Qibo Mo and Changyou Wang equally contributed to this article.
} 
Thinopyrum ponticum (Podp.) Z.-W. Liu \& R.-C. (syn. Agropyron elongatum (Host) Beauv., Lophopyrum ponticum (Podp.) A. Löve, and Elytrigia elongata (Host) Nevski) has been widely used in wheat breeding ( $\mathrm{Li}$ and Wang 2009). Massive disease-resistant genes from Th. ponticum have been transferred into wheat, such as soil-borne diseases common root rot (Li et al. 2004), wheat streak mosaic virus (WSMV), wheat curl mite (WCM) (Chen et al. 2002) and Cephalosporium stripe (Cai et al. 1998) and several multi-resistant wheat-Th. ponticum partial amphiploids were developed, such as Xiaoyan693, Xiaoyan7631, Xiaoyan7430, Xiaoyan68 and Xiaoyan784 (Li et al. 1985). Xiaoyan784 contains 10 A-chromosomes, 14 B-chromosomes, 12 D-chromosomes, 4 A-D translocation chromosomes and 2 A-B translocation chromosomes according to Zheng et al. (2014).

Since multivalent formation was reported in Th. ponticum chromosomes (Muramatsu 1990) and wheat-Th. ponticum hybrids during meiosis, the genomic composition of Th. ponticum has not been clarified yet. Chen et al. (1998) suggested that the genomic composition of Th. ponticum was $\mathrm{JJJ}^{\mathrm{s}} \mathrm{J}^{\mathrm{s}} \mathrm{J}^{\mathrm{s}}$, in which the $\mathrm{J}$ genome of Th. ponticum was closely related to the $\mathrm{J}$ genome of the diploid Th. bessarabicum and the $\mathrm{J}^{\mathrm{s}}$ genome was a modified J genome of unknown origin characterized by the presence of an St (P. spicata) genome-specific hybridization signal near the centromere. However, Zhang et al. (1996) presumed that Th. ponticum consisted of two basic genomes St and E, shown by the formula $\mathrm{StStE}^{\mathrm{e}} \mathrm{E}^{\mathrm{b}} \mathrm{E}^{\mathrm{x}}$, with $\mathrm{E}^{\mathrm{e}}$ from Th. elongatum, $\mathrm{E}^{\mathrm{b}}$ from Th. bessarabicum and $\mathrm{St}$ from Pseudoroegneria species, respectively.

Because of the efficiency and high accuracy, genomic in situ hybridization (GISH) and fluorescence in situ hybridization (FISH) are employed continuously to detect the alien chromosomes or introgression segments transferred into the wheat backgrounds. Diverse functional molecular markers, especially genome-specific markers, were applied to check the targeted alien chromosome, chromosome segments or genes introduced to common wheat (Wang et al. 2010). Therefore, it is convenient to characterize the constitution of the progeny from wheat and its wild relatives by combining those techniques.

In the present study, a) a novel wheat-Th. ponticum 5St (5A) disomic substitution line ES-7 was developed, which was derived from common wheat cultivar Abbondanza and wheat-Th. ponticum partial amphiploid line Xiaoyan784. It was revealed that the introduction of new stripe rust resistance gene(s) from Th. ponticum 5St chromosomes into wheat, which can be used as the bridge material to incorporate the gene into the wheat genome through chromosome translocation. b) the chromosome constitution of this substitution line was characterized, based on mitotic and meiotic cytogenetics and FISH and GISH. c) 5St specific EST-SSR markers were developed and characterized.

\section{Materials and Methods}

\section{Materials}

Materials included Th. ponticum $(2 n=10 x=70)$, Th. elongatum $(2 n=2 x=14)$, Th. bessarabicum $(2 n=2 x=14)$, tetraploid $P$. spicata $(2 n=4 x=28)$, Triticum aestivum cultivar Chinese Spring (CS), Abbondanza, wheat-Th. ponticum partial amphiploid line 
Xiaoyan784, and the substitution line ES-7. All plant materials were provided by the College of Agronomy, Northwest A \& F University. Huixianhong (HXH) was employed as the susceptible controls in the stripe rust resistance tests at the adult stage in the field.

\section{Cytological identification}

Root tips and young spikes were sampled in the field at appropriate stage, and transferred into the Carnoy's fixative fluid (3:1 ethanol-acetic acid mixture and 6:3:1 ethanol-chloroform-acetic acid mixture, respectively) for at least $48 \mathrm{~h}$. The root tips were tinted by $1 \%$ $(\mathrm{w} / \mathrm{v})$ aceto-carmine solution for $1-2 \mathrm{~h}$ and squashed in $45 \%(\mathrm{v} / \mathrm{v})$ acetic acid. The appropriate anthers contain metaphase I cells were squashed in $1 \%$ aceto-carmine solution (Li et al. 2014). The cell with complete chromosomes complements were photographed by an Olympus BX-43 microscope (Japan) equipped Photometrics SenSys CCD camera.

\section{GISH and FISH}

Seeds were germinated in the dark at $23{ }^{\circ} \mathrm{C}$ until roots reached $1-2 \mathrm{~cm}$. The root tips were removed and pretreated in nitrous oxide with $0.8-1.0 \mathrm{MPa}$ for $2 \mathrm{~h}$, after that it were placed in ice, submerging in acetic acid, for $10 \mathrm{~min}$, and then stored in $70 \%$ ethanol at $-20{ }^{\circ} \mathrm{C}$ for later use. The treated root tips were digested in $2 \%$ cellulase mingled $1 \%$ pectinase at $37{ }^{\circ} \mathrm{C}$ for $52-58 \mathrm{~min}$ (different digestion time in accordance with difference between materials); the slides were then prepared using the drop technique as Han et al. (2004).

The total genomic DNA of common wheat Chinese Spring (CS) and Th. ponticum, Th. elongatum, Th. bessarabicum, tetraploid P. spicata, which were used for GISH blocks and probes, respectively, were isolated from seedling leaves using a modified CTAB method (Doyle 1987) and purified by using chloroform. The total genomic DNA of Th. ponticum, Th. elongatum, Th. elongatum, Th. bessarabicum, tetraploid P. spicata as probe was labeled with a Dig-Nick Translation Mix (Roche, Germany). The sheared $\left(121^{\circ} \mathrm{C}\right.$, $5 \mathrm{~min}$ ) genomic DNA of CS was used as blocking DNA and the ratio of blocking DNA and probe is 330:1. The GISH procedure was performed as described in Yang et al. (2014) with minor modifications. Oligonucleotide probes Oligo-pSc119.2 and Oligo-pTa535, with 5' end-labelled by 6-carboxyfluorescein (6-FAM) or 6-carboxy tetramethylrhodamine (Tamra) were synthesized by Shanghai Invitrogen Biotechnology Co., Ltd. (Shanghai, China), could be used to identify the whole set of wheat chromosomes by FISH analysis, the steps of labeling probe and in situ hybridization were according to Tang et al. (2014). Fluorescent signals were viewed and photographed by an Olympus BX53 (Japan) equipped Photometrics SenSys CCD camera DP 80.

\section{EST analysis}

EST markers (http://wheat.pw.usda.gov/SNP/new/pcr_primers.shtml) of wheat chromosomes were all synthesized in AuGCT DNA-SYN Biotechnology Co., Ltd (Beijing, China). These markers were used to further detect the introduced alien chromosomes in the 
wheat-Th. ponticum disomic substitution line ES-7. The volumes of polymerase chain reaction (PCR) assays were $10 \mu \mathrm{l}$, containing $0.1 \mu \mathrm{l}$ of Taq DNA polymerase $(2.5 \mathrm{U} / \mu \mathrm{l})$ (TAKARA, Japan), $0.5 \mu \mathrm{l}$ of each primer, $0.8 \mu \mathrm{l}$ of dNTP mixture $\left(\mathrm{Mg}^{2+}\right)(2.5 \mathrm{mmol} / \mathrm{L})$ (TAKARA, Japan), $1.0 \mu \mathrm{l}$ of DNA template $(40-100 \mathrm{ng} / \mu \mathrm{l}), 1.0 \mu \mathrm{l}$ of $10 \times$ Taq buffer

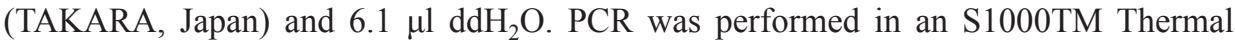
Cycler (Bio-Rad, California, USA) using the following program: initial denaturation at $94{ }^{\circ} \mathrm{C}, 3 \mathrm{~min}$, followed by 35 cycles at $94{ }^{\circ} \mathrm{C}, 30 \mathrm{~s}$, at $50-60{ }^{\circ} \mathrm{C}$ (based on the primer information from the GrainGenes database), $45 \mathrm{~s}$, at $72{ }^{\circ} \mathrm{C}, 50 \mathrm{~s}$ and a final extension step at $72{ }^{\circ} \mathrm{C}, 10 \mathrm{~min}$ before cooling to $4{ }^{\circ} \mathrm{C}$. The PCR products of EST-STS markers were separated in $8 \%$ non-denaturing polyacrylamide gel and visualized with silver staining.

\section{Disease resistance and agronomic trait evaluation}

A mixture of Puccinia striiformis f. sp. tritici (Pst) races CYR32, CYR33 was used to evaluate resistance to stripe rust at the adult stage. Common wheat cv. Abbondanza, Xiaoyan784, the substitution line ES-7 and the susceptible control HXH was separately tested in the field at the College of Agronomy, Northwest A \& F University.

When HXH were fully infected after the artificial inoculation, the reactions to the mixed Pst races were ranked according to a previously published method, the infection types (IT) scores of wheat stripe rust at adult stage was assessed on a scale from $0-4$, as follows: 0,0 ; and 1 were considered to be resistant, 2 was recorded to be moderately resistant, 3 and 4 was assessed to be moderately susceptible and susceptible, respectively (Ma et al. 1995).

Morphological traits of line ES-7 and its parents, common wheat cv. Abbondanza, Xiaoyan784, i.e. plant height, spike length, spikelet numbers and grains per spike were all sampled randomly and investigated.

\section{Results}

\section{Morphology and cytological characterization}

ES-7 was derived from the progeny of wheat cv. Abbondanza and wheat-Th. ponticum partial amphiploid Xiaoyan784. Mitotic and meiotic configurations of line ES-7 showed that there were 42 chromosomes in root tip cells (RTCs) and 21 II in pollen mother cells (PMCs) (Fig. S1*). The chromosome pairing behavior in PMCs was assayed during metaphase I, while no chromosomes was lagged at anaphase I. Therefore, line ES-7 revealed a highly cytological stability.

The plant height, spike length, spikelet numbers and kernels per spike of Xiaoyan784, Abbondanza and ES-7 were shown (Table S1). The plant height of ES-7 was definitely shorter than both parents, and the per spike grains of ES-7 was less. The spikelet numbers of ES-7 were closely resembled to Abbondanza and Xiaoyan784. The spike length of

*Further details about the Electronic Supplementary Material (ESM) can be found at the end of the article. 
ES-7 and Abbondanza showed high similarity, which was significantly shorter than Xiaoyan 784 .

\section{Stripe rust resistance evaluation}

The reactions of adult plants to the mixed Pst races (CYR32 and CYR33) were tested in the field. Wheat parent cv. Abbondanza and control variety $\mathrm{HXH}$ were susceptible, while Xiaoyan784 was almost immune to these two races, meanwhile ES-7 also performed highly resistance to stripe rust at the adult stage (Table S2, Fig. S2). The results indicated that the stripe rust resistance of ES-7 was inherited from Xiaoyan784, which traced to Th. ponticum.

\section{GISH and FISH analysis}

The total genomic DNA of Th. ponticum was used as probes and that of wheat CS were used as block, Mitotic GISH were conducted to identify the introduced chromosomes from Th. ponticum into ES-7. Two green signals were clearly, which means that these two chromosomes in ES-7 were from Th. ponticum (Fig. 1A). And then, the DNA of Th. elongatum, Th. bessarabicum and P. spicata were used as probes, respectively, while the green signals only appear in the condition using the DNA of P. spicata (the results that using the DNA of Th. elongatum, Th. bessarabicum are not shown). These results of somatic cells revealed that ES-7 had two alien chromosomes, with clear hybridization signals, from P. spicata and namely from St genome (Fig. 1B).

To further determine which wheat chromosomes were replaced by Th. ponticum in ES-7, FISH analysis was performed. Oligo-pTa535 and Oligo-pSc119.2 were used to hybridize with ES-7, Abbondanza and Xiaoyan784 mitotic chromosomes by multi-color FISH (Fig. 2, Fig. S3). According to the standard FISH karyotype of CS made by Tang et al. (2014), common wheat chromosomes 5A were missing in line ES-7.

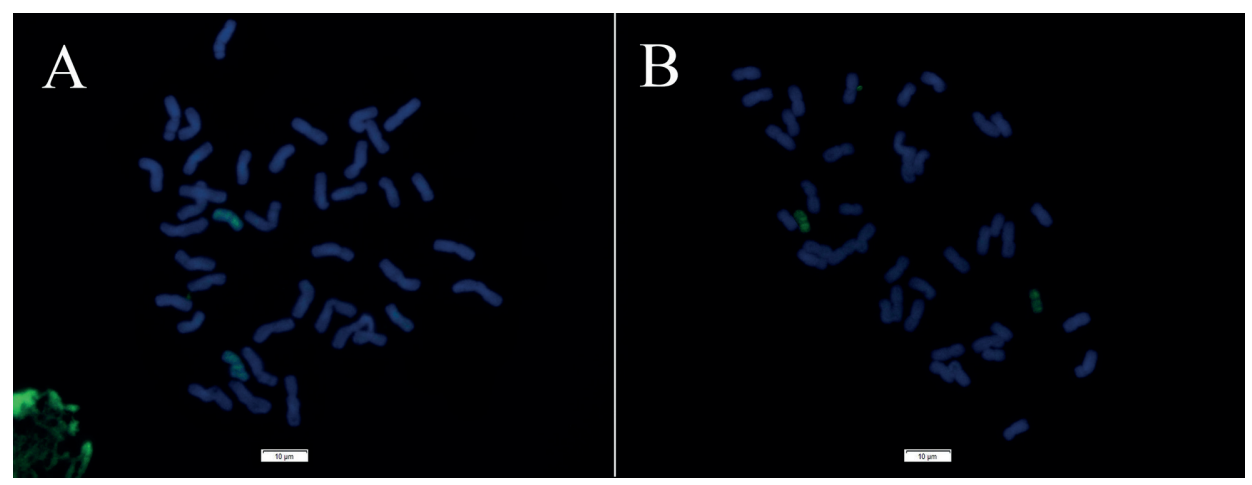

Figure 1. Genomic in situ hybridization (GISH) analysis of ES-7 using Th. ponticum genomic DNA (A: green) and Pseudoroegneria genomic DNA (B: green) as probe, respectively, and CS genomic DNA as block in the ratio of 330:1. Chromosomes were counterstained with DAPI (blue) 


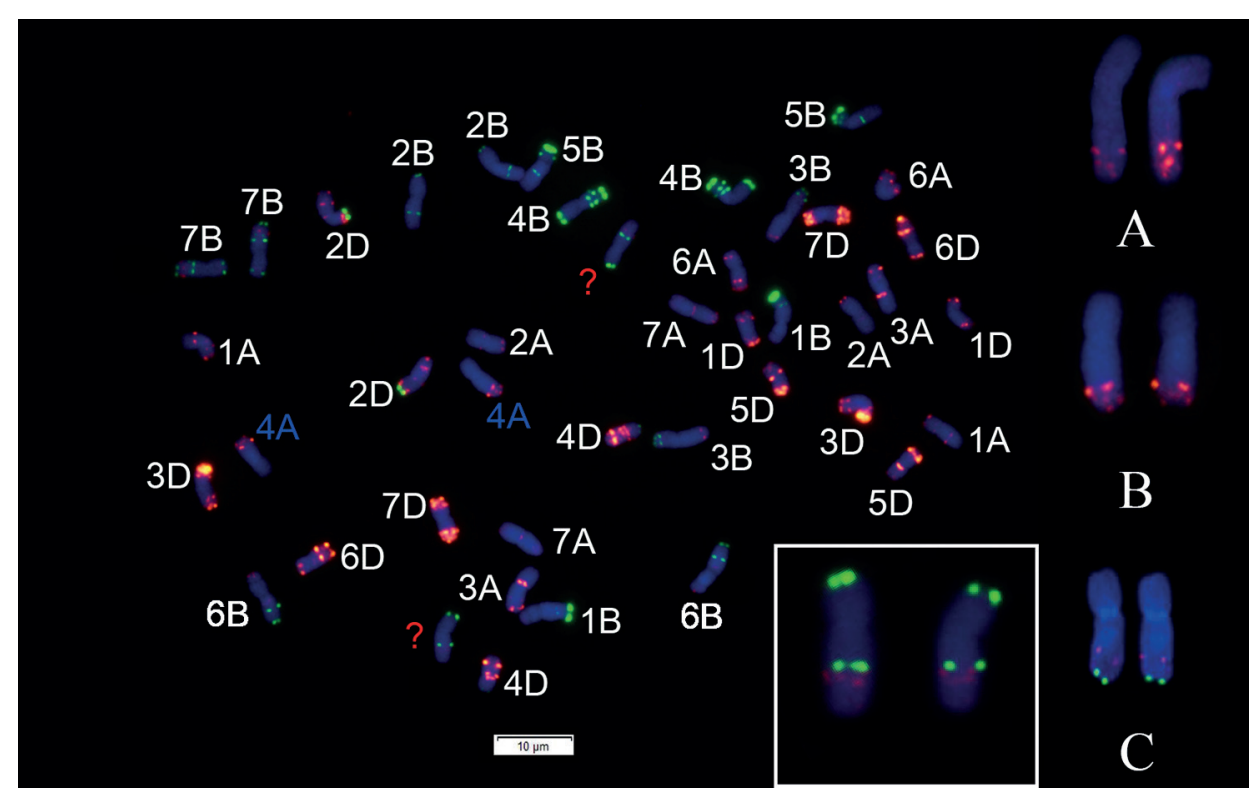

Figure 2. Result of FISH using Oligo-pTa535 (red), Oligo-pSc119.2 (green) and chromosomes were counterstained with DAPI (blue) of ES-7, two interrogations in red show the alien chromosomes (which, are supposed to be $5 \mathrm{St}$, has been cut out and magnified in the bottom right with white square) in ES-7; 4A in Abbondanza (A), 4A in ES-7 (B) and 4A in CS made by Tang et al. (2014) (C) has been cut out and magnified in the right side. Compared to 4A of CS, two green signals in long arm disappeared in Abbondanza and ES-7

Furthermore, two chromosomes, share a similar FISH karyotype and this karyotype was distinct from common wheat chromosomes, were tracked (Fig. 2), which were supposed to be a pair of alien chromosomes from Xiaoyan784, namely, from Th. ponticum and $P$. spicata. Moreover, there were some small alterations between the FISH karyotypes of ES-7, Xiaoyan784 and Abbondanza. ES-7 and Abbondanza lack two green signals (Oligo-pSc119.2) in the long arm of the 4A chromosome, in comparison with published FISH karyotype, and this difference indicated that the 4A in ES-7 was derived from Abbondanza. This occurrence may result from the quite complex genotypic milieu of Xiaoyan784 and Abbondanza.

\section{Molecular marker analysis}

Expressed sequence tag (EST) markers were used to identify which chromosome of Th. ponticum replaced 5A in ES-7. After testing 66 EST molecular markers, two markers (BE482522 and BG262826) (Table S3), which were located on the fifth homoeologous groups of wheat chromosomes, amplified the specific bands of Th. ponticum and P. spicata in ES-7 and Xiaoyan784 (Fig. 3). The result showed that the substitution line possessed a wheat background while it also amplified unique bands from Th. ponticum and P. spicata, which was consistent with the analysis of cytogenetic and GISH. Thus, the 


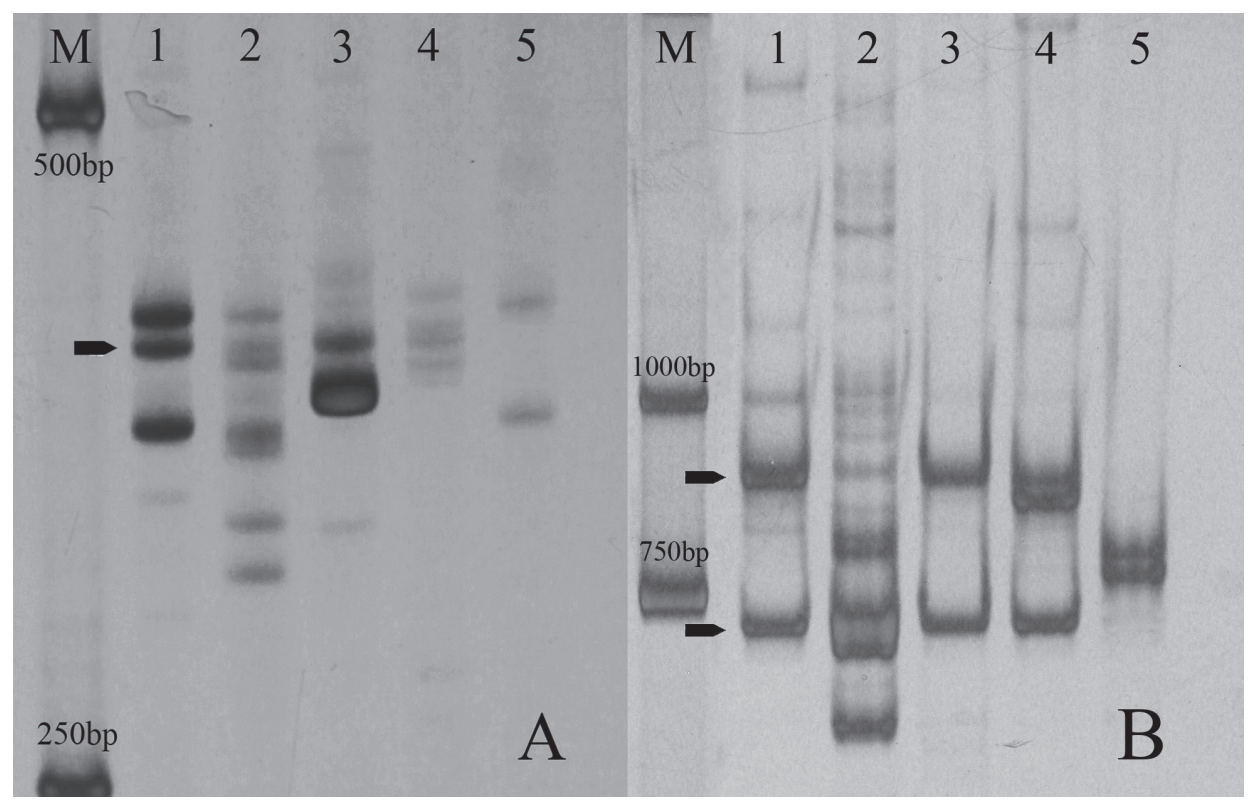

Figure 3. Eight percent non-denaturing polyacrylamide gel electrophoretic analysis of ES-7 A is primer BG262826 and B is primer BE482522. M: DL2000 (Takara) 1: ES-7, 2: Th. ponticum, 3: P. spicata, 4: Xiaoyan 784, 5: Abbondanza. The arrows show the polymorphic bands

alien chromosomes may source from fifth homoeologous groups substituting $5 \mathrm{~A}$ chromosomes of common wheat and two markers (BE482522 and BG262826) can be specific to detect DNA from Th. ponticum and P. spicata.

\section{Discussion}

In summary, a new wheat-Th. ponticum disomic substitution line ES-7 was spontaneously produced from the progeny of a cross between Xiaoyan784 and Abbondanza. This alien disomic substitution line ES-7 was verified by GISH, FISH and EST molecular markers analysis, and the alien chromosomes were most likely to be 5St chromosome. Meanwhile, FISH analysis demonstrated the pair of common wheat chromosomes 5A were absent in ES-7. Therefore, ES-7 was a new 5St (5A) substitution line. Since the selffertility of ES-7 was as high as 91.0\%, we considered that all of the chromosomes in ES-7 accurately paired and separated. Furthermore, wheat-Th. ponticum disomic substitution line ES-7 obtained a significant resistance to stripe rust at adult stage, which could contribute to a higher yield cultivar without this kind of resistance. These traits overall revealed that this new wheat-Th. ponticum disomic substitution line ES-7 can be a better bridge material in wheat breeding than Xiaoyan 784 or other wheat-Th. ponticum partial amphiploids due to relatively similar chromosomal composition with common wheat, especially in culturing new variety with strong stripe rust resistance. 
Th. ponticum was considered as a superior source of resistance to wheat rust (Yin et al. 2006; Li and Wang 2009) and some rust resistance genes have been reported, such as Lr19 (Sarma and Knott 1966), Sr24 (Jiang et al. 1994), Sr25 (Friebe et al. 1994), Sr26 (Friebe et al. 1994) and so on. It was reported that $6 \mathrm{~J}^{\mathrm{s}}$ of Th. ponticum contained relevant genes (Hu et al. 2011). As it was reported that Xiaoyan 784 was highly resistant to all races of stem-rust (Zheng et al. 2014), it is worth noticing that Th. ponticum chromosomes contained novel stripe rust resistance genes and it can be expressed in the wheat back grounds. ES-7 also presented high stripe rust resistance. Accordingly, we supposed that the 5St also had gene(s) that can help wheat from being destroyed by stripe rust. However, there was no systematical study in Th. ponticum. Thus, novel gene(s) relating to stripe rust may be found in Th. ponticum.

Repetitive sequences, pSc119.2 and pTa-535 were usually used as probes in FISH analysis to distinguish wheat A-, B-, and D-genome chromosomes (Pedersen and Langridge 1997; Komuro et al. 2013; Tang et al. 2014). Thus, these two probes proved the absence of $5 \mathrm{~A}$ clearly in this study, which provided facility in the research. However, these probes displayed some small alterations, such as 4A in ES-7, Xiaoyan784 and Abbondanza, in karyotypes between different verities, and this phenomenon may due to two reasons: 1) complex genotypic milieu of parents resulted in some differences in repetitive sequences where the probes hybridizing, so that some signals showed absence or appearance. 2) Introgression that is happening in hybridizing between the wheat and its relative. GISH could detect some chromosome segments coming from non-wheat chromosome. Thus, we did suppose that the main reason that the small alterations existing between the karyotype of Xiaoyan784, Abbondanza, ES-7 and CS was complexity of genotypic milieu.

\section{Acknowledgements}

This research was supported by the scientific research program of Shaanxi province of China (2015KTZDNY01-01-02 and 2016NY-031), the National Key Technology R\&D Program of China (2013BAD01B02-6), and Zhongying Tang Breeding Foundation of Northwest A\&F University.

\section{Author contributions}

W. J. and C. W. designed the experiments; Q. M., C. W., C. C. and Y. W. performed the experiments; Q. M., C. W., H. Z. and X. L. analyzed the data; Q. M., C. W. and W. J. wrote the paper.

\section{Disclosure of potential conflict of interest}

The authors declare no conflict of interest. 


\section{References}

Cai, X., Jones, S.S., Murray, T.D. 1998. Molecular cytogenetic characterization of Thinopyrum and wheatThinopyrum translocated chromosomes in a wheat-Thinopyrum amphiploid. Chromosome Res. 6:183-189.

Chen, Q., Conner, L.R., Ahmad, F., Laroche, A., Fedak, G., Thomas, B.J. 2002. Molecular characterization of the genome composition of partial amphiploids derived from Triticum aestivum $\times$ Thinopyrum ponticum and T. aestivum $\times$ Th. intermedium as sources of resistance to wheat streak mosaic virus and its vector, Aceria tosichella. Theor. Appl. Genet. 97:1-8.

Chen, Q., Conner, L.R., Laroche, A., Thomas, J.B. 1998 Genome analysis of Thinopyrum intermedium and Thinopyrum ponticum using genomic in situ hybridization. Genome 41:580.

Doyle, J. 1987. A rapid DNA isolation procedure for small quantities of fresh leaf tissue. Phytochem. Bull. 19:11-15.

Friebe, B., Jiang, J., Knott, R.D., Gill, S.B. 1994. Compensation indices of radiation-induced wheat-Agropyron elongatum translocations conferring resistance to leaf rust and stem rust. Crop Sci. 34:400-404.

Han, F., Liu, B., Fedak, G., Liu, Z. 2004 Genomic constitution and variation in five partial amphiploids of wheat-Thinopyrum intermedium as revealed by GISH, multicolor GISH and seed storage protein analysis. Theor. Appl. Genet. 109:1070-1076.

Hu, L.J., Li, G.R., Zeng, Z.X., Chang, Z.J., Liu, C., Yang, Z.J. 2011. Molecular characterization of a wheatThinopyrum ponticum partial amphiploid and its derived substitution line for resistance to stripe rust. J. Appl. Genet. 52:279-285.

Huerta-Espino, J., Singh, R.P., German, S., McCallum, B.D., Park, R.F., Chen, W.Q., Bhardwaj, S.C., Goyeau, H. 2011. Global status of wheat leaf rust caused by Puccinia triticina. Euphytica 179:143-160.

Jiang, J., Friebe, B., Gill, B.S. 1994. Chromosome painting of Amigo wheat. Theor. Appl. Genet. 89:811.

Komuro, S., Endo, R., Shikata, K., Kato, A. 2013. Genomic and chromosomal distribution patterns of various repeated DNA sequences in wheat revealed by a fluorescence in situ hybridization procedure. Genome 56:131-137.

Li, H., Wang, C., Fu, S., Guo, X., Yang, B., Chen, C., Zhang, H., Wang, Y., Liu, X., Han, F., Ji, W. 2014. Development and discrimination of 12 double ditelosomics in tetraploid wheat cultivar DR147. Genome 57:89-95.

Li, H., Wang, X. 2009. Thinopyrum ponticum and Th. intermedium: the promising source of resistance to fungal and viral diseases of wheat. J. Genet. Genomics 36:557-565.

Li, H.J., Arterburn, M., Jones, S.S., Murray, T.D. 2004. A new source of resistance to Tapesia yallundae associated with a homoeologous group 4 chromosome in Thinopyrum ponticum. Phytopathol. 94:932-937.

Li, Z.S., Bong, S., Chen, S.Y., Zhong, G.C., Mu, S.M. 1985. Wheat Wide Hybridization. Chinese Scientific Press. Beijing, China.

Ma, H., Singh, R.P., Mujeeb-Kazi, A. 1995. Suppression/expression of resistance to stripe rust in synthetic hexaploid wheat (Triticum turgidum $\times$ T. tauschii). Euphytica 83:87-93.

McIntosh, R.A. 2003. Catalogue of gene symbols for wheat. Cereal Res. Commun. 31:69-71.

Muramatsu, M. 1990. Cytogenetics of decaploid Agropyron elongatum (Elytrigia elongata) $(2 n=70)$. I. Frequency of decavalent formation. Genome 33:811-817.

Pedersen, C., Langridge, P. 1997. Identification of the entire chromosome complement of bread wheat by twocolour FISH. Genome 40:589-593.

Sarma, D., Knott, D.R. 1966. The transfer of leaf-rust resistance from Agropyron to Triticum by irradiation. Can. J. Genet. Cytol. 8:137-143.

Tang, Z., Yang, Z., Fu, S. 2014. Oligonucleotides replacing the roles of repetitive sequences pAs1, pSc119.2, pTa-535, pTa71, CCS1, and pAWRC.1 for FISH analysis. J. Appl. Genet. 55:313-318.

Wang, R., Larson, S., Jensen, K. 2010. Analyses of Thinopyrum bessarabicum, T. elongatum, and T. junceum chromosomes using EST-SSR markers. Genome 53:1083-1089.

Yang, X., Wang, C., Chen, C., Zhang, H., Tian, Z., Li, X., Wang, Y., Ji, W. 2014. Chromosome constitution and origin analysis in three derivatives of Triticum aestivum-Leymus mollis by molecular cytogenetic identification. Genome 57:583-591. 
Yin, X., Shang, X., Pang, B., Song, J., Cao, S., Li, J., Zhang, X. 2006. Molecular mapping of two novel stripe rust resistant genes YrTp1 and YrTp2 in A-3 derived from Triticum aestivum $\times$ Thinopyrum ponticum. Agr. Sci. China. 5:483-490.

Zhang, X., Dong, Y., Wang, R.R.C. 1996. Characterization of genomes and chromosomes in partial amphiploids of the hybrid Triticum aestivum $\times$ Thinopyrum ponticum by in situ hybridization, isozyme analysis, and RAPD. Genome 39:1062-1071.

Zheng, Q., Lv, Z., Niu, Z., Li, B., Li, H., Xu, S.S., Han, F., Li, Z. 2014. Molecular cytogenetic characterization and stem rust resistance of five wheat-Thinopyrum ponticum partial amphiploids. J. Genet. Genomics 41:591-599.

\section{Electronic Supplementary Material (ESM)}

Electronic Supplementary Material (ESM) associated with this article can be found at the website of CRC at http://www.akademiai.com/content/120427/

Electronic Supplementary Table S1. Agronomic traits of the alien substitution line ES-7 and its parents

Electronic Supplementary Table S2. Evaluation of the disease resistance to stripe rust of Th. ponticum, Abbondanza, Xiaoyan784 and ES-7

Electronic Supplementary Table S3. Two specific markers on the fifth homoeologous groups

Electronic Supplementary Figure S1. Mitotic (A $2 n=42)$, meiotic I (B $2 n=21$ II) and anaphase I $(\mathrm{C} 2 n=21+21)$ chromosome characteristics of ES-7

Electronic Supplementary Figure S2. Stripe rust tests of Abbondanza (A), Xiaoyan784 (B) and ES-7 (C) and $\mathrm{HXH}(\mathrm{D})$ at adult stages

Electronic Supplementary Figure S3. Result of FISH using Oligo-pTa535 (red), Oligo-pSc119.2 (green) and chromosomes were counterstained with DAPI (blue) of A: Xiaoyan784 the lower cases from a to $\mathrm{g}$ in red indicate these chromosomes are different from the standard karyotype, and the $1 \mathrm{~B}$ in blue show the chromosomes 1B which have small alterations from the common one; B: Abbondanza the 4A in blue show the chromosomes $4 \mathrm{~A}$ which have small alterations from standard karyotype 